RASĀYAN J. Chem.

Vol. 13 | No. 3 |1417-1423| July - September | 2020

ISSN: 0974-1496 | e-ISSN: 0976-0083 | CODEN: RJCABP

RJC http://www.rasayanjournal.com http://www.rasayanjournal.co.in

\title{
THE STUDY OF PHYSICAL AND CHEMICAL PROPERTIES OF WATER-SOLUBLE POLYMER REAGENTS AND THEIR COMPATIBILITY WITH ANTIBIOTICS
}

\author{
Erzhan Dzhakipbekov ${ }^{1}$, Saule Sakibayeva ${ }^{1}$, Nagima Dzhakipbekova ${ }^{2}$, Botagoz \\ Tarlanova $^{3}$, Guzaliya Sagitova ${ }^{1}$ and Zhadra Shingisbayeva ${ }^{4}$ \\ ${ }^{1}$ M. Auezov South Kazakhstan State University/ Department of Oil Refining and Petrochemistry, \\ Tauke Khan av.5, 160000, Shymkent, Kazakhstan \\ ${ }^{2}$ M. Auezov South Kazakhstan State University/ Department of Chemistry and Fundamentals of \\ Chemical Technology, Tauke Khan av.5, 160000, Shymkent, Kazakhstan \\ ${ }^{3}$ South Kazakhstan Medical Academy / Department Technology of Pharmaceutical Production, \\ Al-Farabi Square 1, Shymkent, Kazakhstan \\ ${ }^{4}$ M. Auezov South Kazakhstan State University / Department of Ecology, Tauke Khan av.5, \\ 160000, Shymkent, Kazakhstan \\ *E-mail: guzal.sagitova@bk.ru
}

\begin{abstract}
The article considers the processes of synthesis of water-soluble polymers HPAA-HP (hydrolyzed polyacrylamide with the modifier hydrogen peroxide), HPAA-MEA (hydrolyzed polyacrylamide with the modifier monoethanolamine), HPAA-TEA (hydrolyzed polyacrylamide with the modifier triethanolamine). The physical and chemical properties of water solutions of water-soluble polymers are investigated. It is shown that they are highmolecular polyelectrolytes. The compatibility of polymers with antibiotics is shown. Synthesized polymers have been proposed as ointments of soft carriers for use with medications. Many known ointment bases are easily exposed to microbial contamination (hydrogenated fats, fatty and vegetable oils, gelatin gels and others). The effect of the antibacterial ointments we are researching is aimed at the destruction of pathogenic microorganisms and products of their vital activity, which are the cause of infectious and inflammatory processes in the wound. Therefore, one of the most important requirements expressed to ointment bases, is resistance to microbial contamination, because the latter can significantly reduce the concentration of antibiotics in ointments, thereby reducing the therapeutic effect of the preparations, and can be the cause of secondary infection of the wound or burn surface. The study of colloidal-chemical properties of aqueous solutions of the studied polymers allowed establishing the relationship between poly electrolyte effects and functional composition, the degree of hydrolysis. The conditions for obtaining HPAA-HP, HPAA-MEA polymers, most clearly showing polyelectrolyte properties were determined based on the research of physical and colloidal - chemical properties, their polyelectrolytic character is revealed.and they belong to high-molecular surfactants they relate to high-molecular surface-active substances.
\end{abstract}

Keywords: Water-soluble Polymer, Soft Carriers, Antimicrobial Activity, Antibiotics, Hydrolyzed polyacrylamide, Ointment, Viscosity.

(C) RASĀYAN. All rights reserved

\section{INTRODUCTION}

The article presents the results of studies of physical and chemical properties of water-soluble polymer reagents and their compatibility with antibiotics. The creation of new polymer reagents fromtheir synthesis in the laboratory to their industrial production as an ointment is a relatively long and expensive process. Therefore, the most promising and justified way is to expand the range of polymer reagents by modifying the already known base samples. We studied HPAA-HP, HPAA-MEA, HPAA-TEA (hydrolyzed polyacrylamide) polymers. Synthesized polymers have been proposed as soft carriers for

Rasayan J. Chem., 13(3), 1417-1423(2020)

http://dx.doi.org/10.31788/RJC.2020.1325709

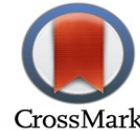


RASĀYAN J. Chem.

Vol. 13 | No. 3 |1417-1423| July - September | 2020

drug transport. To obtain reproducible results, strict standardization of experiments is necessary. The rate of solutions diffusion in agar depends on the chemical nature of the antibiotic, the composition, $\mathrm{pH}$ of the agar medium, the buffer in which the working solutions of the standard and the test material are prepared, the temperature and incubation time. Therefore, when determining the concentration of antibiotics and maintenance of their antibacterial activity in test samples we should select the conditions for culturing the test culture, the optimal composition and $\mathrm{pH}$ of the medium, buffer solutions, ensuring the maximum diffusion of the solutions of antibiotic into the medium and the sharpness of the zones outlines. The determination is carried out according to the scheme common to all antibiotics. ${ }^{1}$

\section{Characteristics of the Studied HPAA-HP, HPAA-MEA Copolymers}

Copolymers of acrylic acid, which are well mixed with water forming a viscous fluid mass, were synthesized based on the Department of chemistry and fundamentals of chemical technology in the M. Auezov SKSU. Their properties and consistency are of interest for the possibility of their use as auxiliary substances in pharmaceutical technology in the preparation of external medical forms.

The preparations have undergone poison control. Based on toxicological studies and analysis of the data obtained, it was concluded that polymers have a slight functional cumulation. They do not show skinblistering and allergic effects. ${ }^{2}$

\section{HPAA- HP, HPAA-MEA}

Hydrolyzed polyacrylamide with the modifier hydrogen peroxide was obtained based on hydrolysis with alkali of polyacrylamide in the presence of a modifier at a temperature of $368 \mathrm{~K}$ for a time of 2-2.5 hours. Hydrolyzed polyacriamide with the monoethanolamine modifier was prepared based on hydrolysis of polyacrylamide with an alkali in the presence of monoethanolamine modifier at a temperature of $368 \mathrm{~K}$ and reaction time of 2 hours.

\section{Polyacrylamide (PAA)}

PAA is granules of irregular shape, yellowish or slightly brown. The mass fraction of the polymer in the product is $50-56 \%$, moisture is $10-14 \%$, and ammonium sulphate is $30-40 \%$. The $\mathrm{pH}$ of $0.1 \%$ water solution is $7-8$. The kinematic viscosity of $0.1 \%$ water solution is not less than $(1.7-2) 10^{-6}{ }^{-3}$

$$
\left[\begin{array}{c}
\mathrm{CH}_{2}-\mathrm{CH}- \\
\mathrm{O} \\
\mathrm{O}=\mathrm{C}-\mathrm{NH}_{2} \mathrm{n}
\end{array}\right]
$$

\section{EXPERIMENTAL}

Conductivity.pH of HPAA-HP, HPAA-MEA, HPAA-TEA polymers solutions were measured on a potentiometer $\mathrm{pH}-340$ with an accuracy of $+/-0.05$ in a thermostatically controlled cell in which the temperature was maintained with an accuracy of $25+/-0.01^{\circ} \mathrm{C}$. Further, the electrical conductivity was determined by the formula $(\chi)$ :

$$
\chi=\frac{\alpha}{R x}
$$

Where, $\alpha$ is the cell constant, it was determined by the formula:

$$
\alpha=0.000147 \mathrm{~m}-1 \mathrm{~cm}-1 \text { at } \mathrm{T}=250^{\circ} \mathrm{C}
$$

The electrical conductivity of GRP HPAA-HP, HPAA-MEA, HPAA-TEA solutions is studied on the device assembled according to the bridge circuit with the frequency of $1000 \mathrm{~Hz}$; voltage was produced by the ZG-6m sound generator. The output voltage is $6 \mathrm{~V}$. The electrical cell has the form of a cylindrical vessel with a capacity of $25 \mathrm{ml}$; electrodes are made of blackened platinum. The arrangement of 
RASĀYAN J. Chem.

Vol. 13 | No. 3 |1417-1423| July - September | 2020

electrodes is vertical. The thermostat of the cell is carried out in a water thermostat. The cell constant $(\mathrm{K})$ at $298 \mathrm{~K}$ is $0.37 . .^{4}$

Specific conductivity $(\chi)$ is calculated according to the formula:

Where, $\mathrm{R}$ - is resistance $\left(\mathrm{Ohm}^{-1}\right)$ of the studied solution;

$$
\chi=\mathrm{K} / \mathrm{R} \times\left(\mathrm{OM}^{-1} \times \mathrm{sm}^{-1}\right),
$$

$\mathrm{K}$ - is cell constant.

Potentiometric titration is carried out on a laboratory $\mathrm{pH}$ meter - millivoltmeter LPM-60 $\mathrm{m}$ with a sensor DL-01 at $298 \pm 0,1 \mathrm{~K}$ in a nitrogen current. The meter is a glass electrode ESL IIG-0.4, and a comparison electrode is calomel electrode. ${ }^{5}$

\section{Microbiological Method of Direct Diffusion into Agar}

To establish the compatibility of HPAA with levomycetin sodium succinate and gentamycin sulfate antibiotics it is necessary to detect the antimicrobial activity of antibiotics, which is determined by diffusion into agar.

The method for carrying out this research is described in detail in the USSR pharmaceutical regulations XI ed., V.II. Because it does not consider some of the features of our experiment (for example, the determination of antimicrobial activity in ointments), we modified the method to be able to apply it in our work.

The method of diffusion into agar is based on comparing the degree of inhibition of the test microbe growth zone with certain concentrations of the antibiotic in the test material with inhibition of its growth zone by the known concentrations of the antibiotic standard. The suppression of the test microbe growth is due to the antibiotic diffusion from the test material into a dense nutrient medium.

To obtain reproducible results, strict standardization of experiments is necessary. The rate of solutions diffusion in agar depends on the chemical nature of the antibiotic, the composition, $\mathrm{pH}$ of the agar medium, the buffer in which the working solutions of the standard and the test material are prepared, and the temperature and incubation time. Therefore, when determining the concentration of antibiotics and maintenance of their antibacterial activity in test samples we should select the conditions for culturing the test culture, the optimal composition and $\mathrm{pH}$ of the medium, buffer solutions, ensuring the maximum diffusion of the solutions of antibiotic into the medium and the sharpness of the zones outlines. The determination is carried out according to the scheme common to all antibiotics. ${ }^{6}$

\section{Spectrophotometric Quantitative Determination of Levomycetin Sodium Succinate in Antibacterial Ointments}

This highly sensitive method of quantitative determination of the drug in solution is described in Pharmaceutical standard-42-737-78.

The optical density of the $0.002 \%$ preparation solution was measured on the SF at a wavelength of 275 $\mathrm{nm}$ compared to water in a sample-holder with a layer thickness of $1 \mathrm{~cm}$. The same measurement was carried out with a $0.002 \%$ solution of a standard sample at a wavelength of $278 \mathrm{~nm}$.

The percentage $(\mathrm{X})$ of levomycetin in the preparation is calculated by the formula:

$$
X=\frac{D+C_{0}+100}{\mathrm{D}_{0}+C},
$$

Where,

D-is the optical density of the test solution

D0- is the optical density of a solution of a standard sample

$\mathrm{C}$-is the concentration of the test solution

$\mathrm{C} 0$ - is the concentration of the solution of the standard sample.

The content of levomycetin in the preparation should be at least $65 \%$. Each flask should contain at least $90 \%$ and not more than $110 \%$ of the amount indicated on the label. ${ }^{7}$

Note: The standard sample is levomycetin (Pharmaceutical Standard for Levomycetin). 
RASĀYAN J. Chem.

Vol. 13 | No. 3 |1417-1423| July - September | 2020

\section{Fractional Sterilization (Tundalization).}

The objects to be sterilized are heated at $30^{\circ} \mathrm{C}$ for 1 hour every 24 hours. In the intervals between heating, the object is kept in a thermostat at a temperature favorable for the spore germination $\left(25-37^{\circ} \mathrm{C}\right)$. Three cycles are enough for all the spores contained in the object to germinate and die with subsequent heating.

\section{The Method of Equilibrium Dialysis According to Kruvchinsky.}

The dialysis device proposed by Kruvchinsky consists of a beaker that places $50 \mathrm{ml}$ of the model medium, a dialysis tube with an internal diameter of $32 \mathrm{~mm}$ and a height of $160 \mathrm{~mm}$. Put $1.0 \mathrm{~g}$ of the test sample of the ointment in the lower end of the tube. Put the tube into a glass with a dialysis medium to a depth of 2 $\mathrm{mm}$. A thermometer for temperature control and a pipette for sampling are put into the glass. The glass is placed in a water bath. Samples have a volume of $2 \mathrm{ml}$.

\section{RESULTS AND DISCUSSION}

The property of HPAA-HP, HPAA-MEA, HPAA-TEA polymers to dissociate in aqueous solution allows us to study the electrical conductivity of aqueous solutions of polymers and to research the ionizability of macromolecules in solution. It is known that electrical conductivity depends on the concentration of polymers. With the increasing concentration of the studied samples of GRP, the specific conductivity increases, and then this dependence is exponential. Thus $\chi_{\text {sp }}$ depends on a ratio of polymers functional groups. ${ }^{8}$

In such systems, it is seen that the distribution of mobile ions between the areas occupied by poly ions and in the surrounding space is not regulated by Donnan balance. The activity coefficients of ions must be compressed at the expense of the existing electrostatic forces, which lead to a decrease in the potential and, accordingly, in their electrical conductivity. Also, the possible formation of ion pairs, associates,chelatesformed due to the hydrogen bonds can take place.

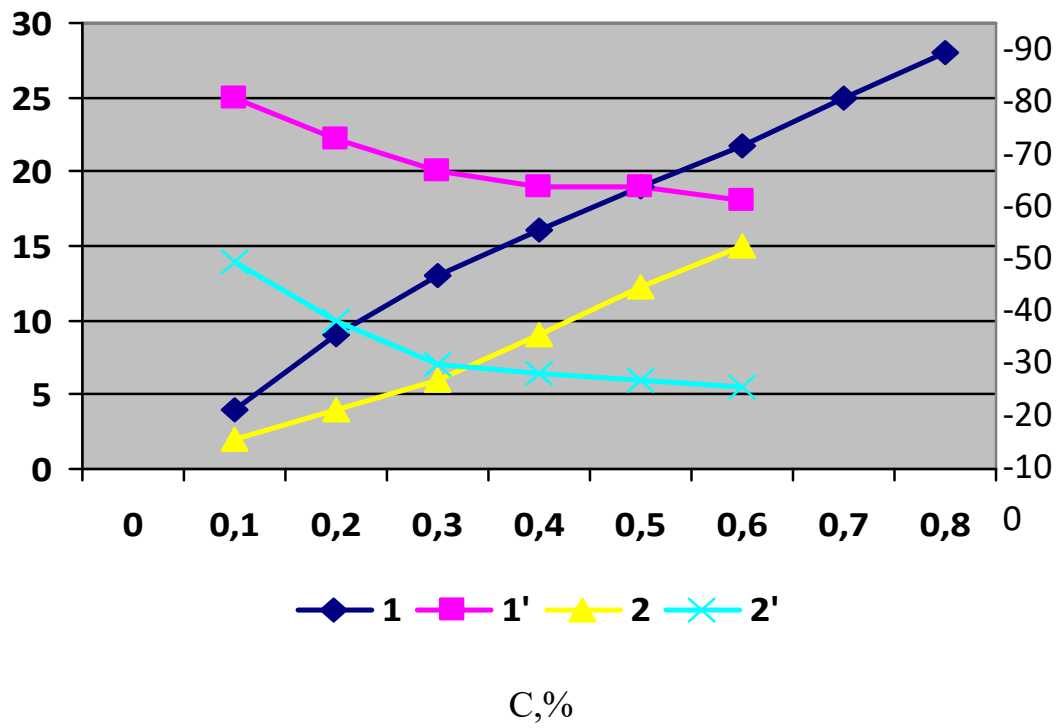

Specific $(1,2)$ and Equivalent $\left(1^{\prime}, 2^{\prime}\right)$ Conductivity of Solutions: 1-HPAA-HP; 2-HPAA-MEA

Fig.-1: Relationship of Specific and Equivalent Conductivity in HPA-PV, HPA-IEA Polymer Solutions

Figure-1 shows that the initial incident portion of the curve corresponds to the region of the complete ionization of functional groups and thermodynamic stability of the system. Straight section parallel to the absciss axis corresponds to the area of structured solutions in which macromolecules are associated. The fracture on the curve can be considered as the beginning of the conformational transformation of macromolecular tangles in HPAA-HP, HPAA-MEA polymer solutions with the possible formation of supramolecular structures in concentrated polymersolutions. 
RASĀYAN J. Chem.

Vol. 13 | No. 3 |1417-1423| July - September | 2020

In all HPAA-HP GRP samples, the pH-q correlation has S-shaped form with inflection points in the acidic (3.5-4.0) and alkaline (8.5-10) areas (Fig.-2). In an acidic medium (pH=3.6-4.2), $\mathrm{COOH}$ groups slightly dissociate and are in a tangled state; when alkali is added, the neutralization of hydrogen ions mainly occurs at higher $\mathrm{pH}$. The neutralization reaction is accompanied by the appearance of charges on the macromolecule, which affects the titration curve and the chain shape.

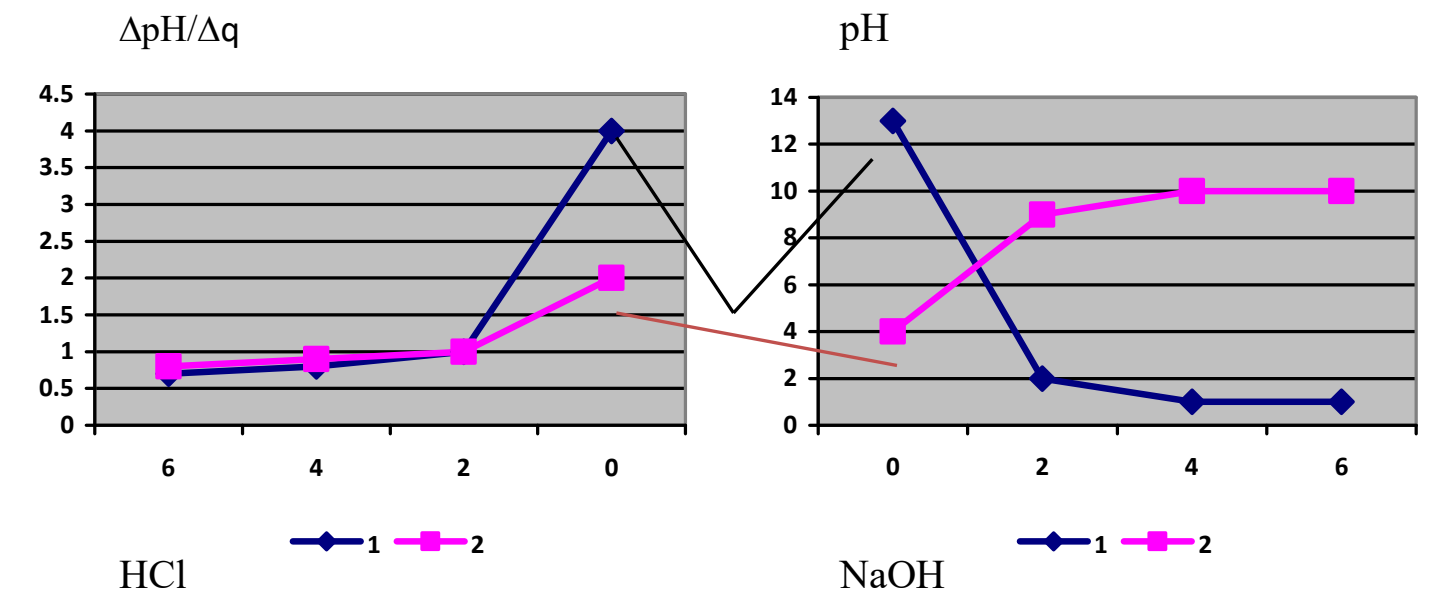

Fig.-2: Integral (1) and Differential (2) Potentiometric Titration Curves of 0.1\% Solution of the HPAA-PV, HPAAIEA Dispersions Mixture

Curves with inflection points in the acidic and alkaline regions indicate the presence of both acidic and basic functional groups.Fully titrated acidic and basic groups correspond to extreme points on the differential curves of potentiometric titration.

For HPAA-HP, HPAA-MEA amphoteric polymers, the titration curve looks more complicated than for polyacids and polybases.

Carboxyl groups of HPAA-HP, HPAA-MEA are titrated in the $\mathrm{pH}$ range=4-9. At $\mathrm{pH}$ below four, hydrochloric acid joins the amide and imide groups to form the salt of hydrochloric acid polyamide, meanwhile $\mathrm{pH}$ changes to three. Earlier it was established that for a polypeptide chain electrolytic interaction of closely spaced groups differs for spiral and tangle-shaped conformations; in the transition spiral-tangle region, the degree of ionization of the macromolecule increases sharply when the $\mathrm{pH}$ of the solution changes. ${ }^{9}$

The electrical conductivity of solutions HPAA-HP, HPAA-MEA (Fig.-3) is studied. An increase in the concentration of HPAA-HP, HPAA - MEA solutions leads to an increase in specific conductivity up to a certain concentration. Then this correlation becomes exponential. This can be explained by either the fact that not all ionogenic groups of the polymer are ionized, or that some factors impede electricity transfer.

\section{Development of the Ointment Base Optimal Composition}

When developing the ointment base optimal composition, $1.0 \mathrm{~g}$ of HPAA polymer was dissolved in different volumes of distilled water: $1 \mathrm{ml}, 2 \mathrm{ml}, 3 \mathrm{ml}, 4 \mathrm{ml}, 5 \mathrm{ml}$ and $6 \mathrm{ml}$.

It was found out that to obtain an ointment base with the necessary consistency, the optimal ratio of HPAA polymer and distilled water was 1: 5 .

The pre-ground and screened HPAA polymer powder was weighed on a BP-1 scale in an amount of $1.0 \mathrm{~g}$, transferred into a mortar and triturated with $5 \mathrm{ml}$ of distilled water, added in parts. As a result of a tenminute stirring, a thick, sticky, gel-like, transparent mass without odor, the yellowish-cream color was obtained. The base is easily applied to the skin when drying it forms a thin protective film on it, easily removable with a cotton swab dipped in water. The base does not spoil underwear and clothes, does not have an irritating effect and does not tighten the skin when it dries. 
RASĀYAN J. Chem.

Vol. 13 | No. 3 |1417-1423| July - September | 2020

Thus, the technology of this synthetic hydrophilic base preparation is extremely simple, does not require special methods of preparation and compliance with a lot of conditions. It is convenient and quick to use.

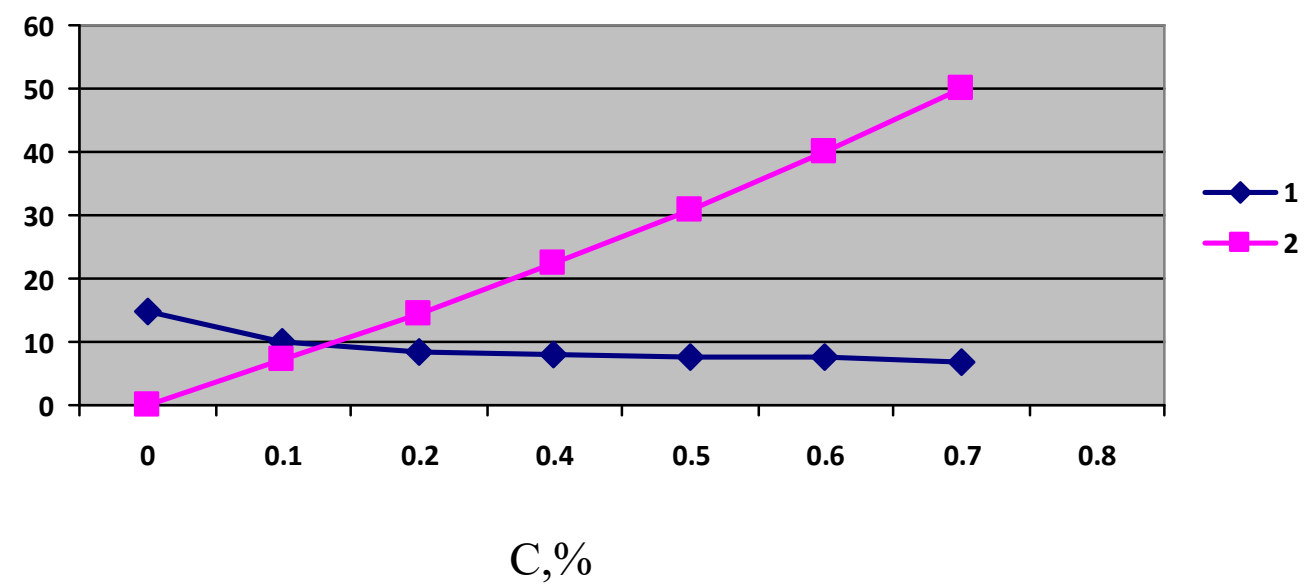

Fig.-3: Correlation of Specific (1) and Equivalent Conductivity (2) of HPA-PV, HPA-IEA Dispersion Mixture Solutions.

\section{Determination of the HPAA New Synthetic Hydrophilic Base of Resistance to Microbial Contamination}

Many known ointment bases are easily exposed to microbial contamination (hydrogenated fats, fatty and vegetable oils, gelatin gels and others). The effect of the antibacterial ointments we are researching is aimed at the destruction of pathogenic microorganisms and products of their vital activity, which are the cause of infectious and inflammatory processes in the wound.

Therefore, one of the most important requirements expressed to ointment bases, is resistance to microbial contamination, because the latter can significantly reduce the concentration of antibiotics in ointments, thereby reducing the therapeutic effect of the preparations, and can be the cause of secondary infection of the wound or burn surface.

In connection with the above, it seems necessary to research this direction, using a microbiological agar method.

For this purpose, $1 \mathrm{~g}$ of HPAA base was added to two tubes containing $4 \mathrm{ml}$ of molten and cooled to $50^{\circ}$ $\mathrm{C}$ nutrient medium. The content of the tube was quickly and thoroughly mixed and transferred to a Petri dish containing $20 \mathrm{ml}$ of a solidified aerated media.

Spread the top layer of agar evenly with a quick rocking of the Petri dish. After solidifying of the medium, the plates were incubated in a thermostat for 5 days at $35^{\circ} \mathrm{C}$.

During this period growth of the bacteria was not detected. Consequently, there are less than 10 bacteria in $1 \mathrm{~g}$ of the polymer base. The permissible limit of microorganisms' content in medicinal forms of local use is the presence of not more than 100 microorganisms per gram of the drug.

\section{CONCLUSION}

1. The study of colloidal-chemical properties of aqueous solutions of the studied polymers allowed establishing the relationship between polyelectrolyte effects and functional composition, the degree of hydrolysis. The conditions for obtaining HPAA-HP, HPAA-MEA polymers, most clearly showing polyelectrolyte properties were determined

2. The research of physical and colloid-chemical properties of polymers revealed 1422heirpolyelectrolyte nature; they relate to high-molecular surface-active substances.

3. Compatibility of HPAA with antibiotics of levomycetin sodium succinate and gentamycin sulfate has been established as well as antimicrobial activity of antibiotics. 
RASĀYAN $J$. Chem.

Vol. 13 | No. 3 |1417-1423| July - September | 2020

\section{REFERENCES}

1. N. Dzhakipbekova, S. Sakibayeva, E. Dzhakipbekov, D. Ahmet, S. Rzabay, A. Issa, A. Kydyralyeva and E. Amantayev, Oriental Journal of Chemistry, 34 (4), 1779(2018), DOI: $10.13005 / \mathrm{ojc} / 3404010$

2. K. J. Febbereitor, Colloid and Polymer Science, 256(5), 490(1978).

3. P. A. Abdurazova, Sh. T. Koshkarbayeva, M. S. Satayev, N. O. Dzhakipbekova and Ye. B. Raiymbekov, Bulletin of the University of Karaganda-Chemistry, 3(95), 45(2019), DOI:10.31489/2019Ch3/45-51

4. R. Kannan, M. Selvambikai, I. Jeena Rajathy and S. Anathi, Rasayan Journal of Chemistry, 10(4), 1213(2017), DOI:10.7324/RJC.2017.1041925

5. P. A. Abdurazova, U. B. Nazarbek, A. A. Bolysbek, N. K. Sarypbekova, G. S. Kenzhibayeva, G. A. Kambarova, M. S. Sataev, Sh. T. Koshkarbaeva, A. B. Tleuova, S. Perni and P. Prokopovich, Colloids and Surfaces A: Physicochemical and Engineering Aspects, 532,115(2017), DOI: 10.1016/j.colsurfa.2017.06.070

6. P.C. Chikezie, C.O. Ibegbulem, F.N. Mbagwu, Research Journal of Phytochemistry, 9(3), 88(2015), DOI:10.3923/rjphyto.2015.88.115

7. N.S. Ncube, A.J. Afolayan, A.L. Okoh, African Journal of Biotechnology, 7(12), 179(2008), DOI: 10.5897/AJB07.613

8. R. A. McBride, J. M. Kelly and D. E. McCormack, Journal of Materials Chemistry, 13, 1196 (2003).

9. T. Lokesh, B. Sarada, K. Swarnalatha, M.A. Kareem, International Journal of Pharmaceutical Sciences and Research, 7(9), 3857(2016), DOI:10.13040/IJPSR.0975-

[RJC-5709/2019] 